\title{
Noninvasive prognostic markers for cardiac death and ventricular arrhythmia in long-term follow-up of subjects with chronic Chagas' disease
}

P.R. Benchimol Barbosa ${ }^{1,2}$

\author{
${ }^{1}$ Seção de Arritmia e Eletrofisiologia, Hospital Universitário Pedro Ernesto, \\ Universidade do Estado do Rio de Janeiro, Rio de Janeiro, RJ, Brasil \\ ${ }^{2}$ Serviço de Métodos Diagnósticos em Cardiologia, Hospital Central Aristarcho Pessoa, \\ Corpo de Bombeiros Militar do Estado do Rio de Janeiro, Rio de Janeiro, RJ, Brasil
}

\section{Correspondence \\ P.R. Benchimol Barbosa Rua Pompeu Loureiro, 36/702 22061-000 Rio de Janeiro, RJ Brasil \\ E-mail: ecgar@yahoo.com}

Research supported by Faculdade de Ciências Médicas, UERJ (No. 5152/2001), Rio de Janeiro, RJ, Brasil.

Received April 28, 2006 Accepted October 9, 2006

\begin{abstract}
The objective of the present study was to investigate clinical, echocardiographic and electrocardiographic (12-lead resting ECG, 24-h ambulatory ECG monitoring and signal-averaged ECG (SAECG)) parameters in subjects with chronic Chagas' disease in a long-term follow-up as prognostic markers for adverse outcomes. Fifty adult outpatients (34 to 74 years old, 31 females) staged according to Los Andes class I, II or III and complaining of palpitation were enrolled in a longitudinal study. SAECG was analyzed in time and frequency domains and the endpoint was a composite of cardiac death and ventricular tachycardia. During a follow-up of $84.2 \pm 39.0$ months, $34.0 \%$ of the patients developed adverse outcomes ( 9 cardiac deaths and 11 episodes of ventricular tachycardia). After optimal dichotomization, in a stepwise multivariate Cox-hazard regression model, apical aneurysm $(\mathrm{HR}=3.7 ; 95 \% \mathrm{CI}=1.2-1.3 ; \mathrm{P}=0.02)$, left ventricular ejection fraction $<62 \%(\mathrm{HR}=4.60 ; 95 \% \mathrm{CI}=1.39-15.24 ; \mathrm{P}=0.01)$ and incidence of ventricular premature contractions $>614$ per $24 \mathrm{~h}$ (hazard ratio $=6.1 ; 95 \% \mathrm{CI}=1.7-22.6 ; \mathrm{P}=0.006$ ) were independent predictors of the composite endpoint. Although a high frequency content in SAECG demonstrated association with the presence of left ventricular dysfunction and myocardial fibrosis, its predictive value for the composite endpoint was not significant. Apical aneurysms, reduced left ventricular function and a high incidence of ventricular ectopic beats over a 24-h period have a strong predictive value for a composite endpoint of cardiac death and ventricular tachycardia in subjects with chronic Chagas' disease.
\end{abstract}

\section{Introduction}

American trypanosomiasis (Chagas' disease) is an anthropozoonosis circumscribed to the American continent and the major
Key words

- Chagas' disease

- Cardiomyopathy

- Noninvasive prognostic assessment

- Long-term follow-up study

- Echocardiography

- Electrocardiography cause of chronic cardiomyopathy due to an immune-mediated response in Latin America. The disease affects over $18,000,000$ people in South America (1) with a mortality rate of 6000/year $(2,3)$, potentially becom- 
ing a significant healthcare-related problem in Europe and the United States due to migration (4). Systemic infection is generally acquired at an early age $(5,6)$, has an insidious and asymptomatic course ranging from 10 to 30 years and progresses to myocardial dysfunction, which occurs in 20-25\% of infected subjects, representing a marker of unfavorable prognosis for cardiac mortality (7-10).

Electrical markers of abnormal ventricular activation $(5,11,12)$ and repolarization $(13,14)$ have been demonstrated to be important predictors of mortality risk in both the general population and in subjects with Chagas' disease. Particularly, the signalaveraged electrocardiogram (SAECG) is a noninvasive method applied to assess the presence of abnormal electrical potentials during ventricular activation (ventricular late potentials), which are considered to be markers of myocardial fibrosis and arrhythmia (15-17). However, information regarding the predictive value of SAECG in subjects with chronic Chagas' cardiomyopathy is limited $(18,19)$. Thus, the objective of the present study was to assess the prognostic value of clinical, echocardiographic, and 12-lead resting ECG, 24-h ambulatory ECG monitoring, and SAECG parameters in subjects with Chagas' disease in a long-term followup.

\section{Material and Methods}

In a longitudinal prospective study, a cohort of 50 consecutive subjects (34 to 74 years old; 31 females) with Chagas' disease followed up for at least 10 years at the cardiomyopathy outpatient clinic of Pedro Ernesto University Hospital, Rio de Janeiro, RJ, Brazil, a tertiary care center, was enrolled from 1995 to 1999. Subjects had been born in endemic regions of the Minas Gerais and Bahia States of Brazil and Chagas' disease was diagnosed on the basis of positive serum tests, hemagglutination cruzipain-
ELISA and indirect immunofluorescence. All subjects were referred to the arrhythmia unit with a complaint of palpitations. At the time of admission, due to the lack of beneficial effects demonstrated thus far, none had received nitroderivative therapy. According to the Los Andes classification (20), subjects were staged into three groups: class I - 14 subjects (group 1), class II - 24 subjects (group 2), and class III - 12 subjects (group 3). Clinical and laboratory data were assessed during a personal interview and by review of medical records. At admission, all subjects were in New York Heart Association (NYHA) functional class I or II, had normal sinus rhythm and normal PR interval. Exclusion criteria at initial enrollment were: any degree of atrioventricular block or non-sinus rhythm, previous documented acute coronary events (unstable angina or myocardial infarction), chronic obstructive pulmonary disease, rheumatic or nonrheumatic valvular heart disease, alcohol addiction, thyroid dysfunction, or abnormal serum electrolytes. A treadmill stress test or a coronary artery angiogram was indicated in selected subjects to exclude concomitant coronary artery disease. World Health Organization and Helsinki Treaty regulations reviewed in Venice (1983) were followed. The study was approved by the local Ethics Committee and all subjects provided written informed consent.

\section{Clinical follow-up}

All subjects have been followed-up by the same team of physicians for the past 10 years. Medical visits have been scheduled at the outpatient clinic at 6-month intervals and medications are prescribed at the discretion of the physician who performed the primary evaluation. Body weight varied $<2 \mathrm{~kg}$ during follow-up, and serum potassium varied from 3.5 to $5 \mathrm{mEq} / \mathrm{L}$. Mild systemic arterial hypertension was observed in $41 \%$ of the patients and was treated with converting 
enzyme inhibitors, diuretics, vasodilators and/or B-blockers at the discretion of the physician who performed the primary evaluation in order to reduce blood pressure levels to less than 140/90 $\mathrm{mmHg}$. The follow-up of the present study was abbreviated according to the endpoint, defined as a composite of either cardiac death or documented episodes of ventricular tachycardia (VT). Subjects were excluded from follow-up after the first documented VT endpoint. All causes of adverse events were ascertained by review of the medical records and by arrhythmic episodes documented by 24-h ambulatory ECG monitoring or 12-lead resting ECG records. The difference between the last and the initial NYHA classes was used to assess progression of subjective symptoms during follow-up.

\section{Echocardiogram}

M-mode and two-dimensional echocardiograms were analyzed by a trained observer blind to the study using an Apogee CX 200 apparatus (ATL, Bothell, WA, USA) with a 4-MHz broadband transducer. Echocardiographic parameters were assessed according to standard procedures of the Section of Echocardiography of the Department of Cardiology, with special care taken to detect left ventricular apical aneurysms (21). The echocardiographic parameters assessed were left ventricular ejection fraction (LVEF) calculated by the Theicholz method, M-mode left ventricular end diastolic diameter (LVEDD), left ventricular end systolic diameter(LVESD), and left atrial diameter, interventricular septal thickness and posterior wall thickness, and the presence of an apical aneurysm. Serial echocardiograms were scheduled at the discretion of the physician who performed the primary evaluation in order to monitor the progression of systolic function during follow-up, which was assessed as the difference between the last and initial estimates of LVEF.

\section{Resting surface ECG and corrected XYZ Frank vectorcardiogram}

For each subject, standard resting 12lead ECGs were recorded in the supine position (with simultaneous 3-lead acquisition) with a Cardimax ECAPS 122000 Compliant Electrocardiograph (Nihon-Kohden Co., Tokyo, Japan) on the same day of blood sample collection. Electrocardiographic abnormalities were classified according to standard criteria for conduction disturbances (intraventricular and atrioventricular), chamber overload, and pathologic $\mathrm{Q}$ waves. The vectorcardiogram was recorded using correctedXYZ Frank leads with 1507c Programmer model Hewlett Packard equipment (Hewlett Packard, Palo Alto, CA, USA) with the subject in the supine position, in a quiet environment, and used to assess the presence of intraventricular conduction disorders and to confirm electrical markers of inactive areas (pathologic Q wave in a 12-lead resting ECG). The electrocardiographic variables assessed were: P-wave duration (on lead II), PR interval (on lead II), QRS complex duration (the longest ventricular duration in precordial leads), presence of bundle branch block and/or left hemi-block, presence of pathological Q waves (defined as the first QRS deflection $>1$-mm deep and $>0.04-\mathrm{ms}$ wide), and left atrial overload ( $\mathrm{P}$-wave duration in lead II $>110 \mathrm{~ms}$ or Morris index in V1 $>4 \mathrm{mV} \cdot \mathrm{ms})$. An independent observer blind to the study analyzed the electrocardiographic $(2 \mathrm{~N}$ and $50 \mathrm{~mm} / \mathrm{s}$ ) and vectorcardiographic records automatically obtained with the electrocardiograph equipment. The ECG was recorded at each clinical visit in order to assess cardiac rhythm and ventricular arrhythmia during follow-up.

\section{Twenty-four-hour ambulatory ECG monitoring}

Twenty-four-hour ambulatory ECG monitoring was performed using a three-channel 
DMS cassette-tape recorder and carefully analyzed using the Del-Mar Avionics StrataScan System (Del Mar Avionics, Irvine, CA, USA) by a trained observer blind to the study in order to assess the presence of ventricular arrhythmia and atrioventricular conduction disturbances. Variables assessed in the 24-h ambulatory ECG were: i) isolated premature ventricular contractions ( $\mathrm{PVC} / 24$ h), ii) coupled ventricular premature complexes (couplets/24 h), iii) polymorphic PVC (PVCs with more than one morphology), and iv) VT episodes (heart rate $>100 \mathrm{bpm}$, QRS duration $>120 \mathrm{~ms}$, and atrioventricular dissociation. Sustained VT was defined as episodes of VT lasting $30 \mathrm{~s}$ or more, with non-sustained VT being characterized by different values. During follow-up, 24-h ambulatory ECG was scheduled from 2 to 5 years in order to assess cardiac rhythm and arrhythmia. All tape recordings were successfully analyzed, and both normal rhythm and arrhythmia episodes were appropriately assessed.

\section{Signal-averaged electrocardiogram}

SAECG was acquired with a Predictor IIc apparatus (ART Inc., Fitchburg, MA, USA) using XYZ Frank orthogonal leads and coherent-averaged up to the noise level of $0.3 \mu \mathrm{V}$. SAECGs were analyzed in both time and frequency domains using a previously reported technique $(22,23)$ by an independent observer blind to the study protocol. In the time domain, SAECG was analyzed on the magnitude vector and parameters were: duration of the filtered ventricular activation (ms), duration of the potentials at the terminal region of ventricular activation below 40 $\mu \mathrm{V}(\mathrm{ms})$, and root-mean-squared voltage of the terminal $40 \mathrm{~ms}$ of ventricular activation $(\mathrm{RMS} 40, \mu \mathrm{V})$. Identification of at least 2 abnormal parameters of 3 defined the presence of ventricular late potentials. In the presence of bundle branch block, ventricular late potentials were present if RMS40 $<14$ $\mu \mathrm{V}$, regardless of filtered QRS duration (24). In the frequency domain, spectral turbulence analysis was carried out using a previously reported technique on a vector magnitude map (25). On the vector magnitude time-frequency map, two-by-two neighbor power spectral segment correlations were deployed in a time series, and the mean and standard deviation were calculated. For each segment, the frequency corresponding to $80 \%$ of the area under the power density function was tracked over the ventricular activation and deployed into a time-series. The mean and the standard deviation of the edge track time-series were analyzed. The correlation between successive power-spectral estimates was limited to the range from 0 to $300 \mathrm{~Hz}$ in order to avoid interference of noise. Using previously defined normality thresholds, identification of at least 2 abnormal parameters of 4 defined the presence of abnormal spectral turbulence.

SAECG parameters were assessed as markers of myocardial fibrosis in the time or frequency domains (23).

\section{Treadmill stress test and coronary artery angiography}

Subjects underwent a treadmill stress test in order to evaluate the presence of coronary artery disease. Five subjects underwent coronary angiography and ventriculography after an inconclusive test and all demonstrated normal coronary artery anatomy. An apical aneurysm was demonstrated in three subjects during ventriculography.

Clinical, echocardiographic, electrocardiographic, 24-h ambulatory ECG, and SAECG variables grouped according to Los Andes classification are presented in Table 1.

\section{Statistical analysis}

Categorical variables are reported as ratio or percentage, and continuous variables as mean $\pm \mathrm{SD}$. Univariate analysis was ap- 
plied to categorical variables by the Yatescorrected chi-square test for contingency tables or Fisher's exact test when appropri- ate. Continuous variables were compared after standard skewness normality assessment by the Student $t$-test or Mann-Whitney test,

Table 1. Clinical, electrocardiographic and echocardiographic parameters of the patients on admission.

\begin{tabular}{|c|c|c|c|c|}
\hline & \multicolumn{3}{|c|}{ Los Andes classification } & \multirow[t]{2}{*}{$\mathrm{P}$} \\
\hline & $\begin{array}{c}\text { Class I } \\
\text { (group } 1 ; \mathrm{N}=14 \text { ) }\end{array}$ & $\begin{array}{c}\text { Class II } \\
\text { (group 2; } \mathrm{N}=24 \text { ) }\end{array}$ & $\begin{array}{c}\text { Class III } \\
\text { (group } 3 ; \mathrm{N}=12)\end{array}$ & \\
\hline \multicolumn{5}{|l|}{ Clinical variables } \\
\hline Age (years) & $52.6 \pm 7.8$ & $51.5 \pm 9.9$ & $49.8 \pm 10.6$ & $>0.05$ \\
\hline Gender (F/M) & $9 / 5$ & $19 / 5$ & $7 / 5$ & $>0.05$ \\
\hline NYHA functional class >1 (\%) & $0 \%$ & $0 \%$ & $33.3 \%$ & 0.001 \\
\hline \multicolumn{5}{|l|}{ Resting 12-lead electrocardiogram } \\
\hline P-wave duration (ms) & $90.0 \pm 17.1$ & $91.3 \pm 17.0$ & $89.2 \pm 22.7$ & $>0.05$ \\
\hline QRS duration (ms) & $80.0 \pm 7.8$ & $127.5 \pm 24.7$ & $121.7 \pm 31.6$ & $<0.001$ \\
\hline Left atrial overload (\%) & $7.1 \%$ & $12.5 \%$ & $16.7 \%$ & $>0.05$ \\
\hline Left bundle branch block (\%) ${ }^{a}$ & $0 \%$ & $16.7 \%$ & $16.7 \%$ & $>0.05$ \\
\hline Right bundle branch block (\%)a & $0 \%$ & $70.8 \%$ & $41.7 \%$ & 0.001 \\
\hline Left anterior hemi-block (\%) & $0 \%$ & $41.7 \%$ & $50.0 \%$ & 0.009 \\
\hline Inactive area (\%) & $0 \%$ & $25.0 \%$ & $41.7 \%$ & 0.03 \\
\hline \multicolumn{5}{|c|}{ M-mode and 2-D mode echocardiogram } \\
\hline $\mathrm{LAD}(\mathrm{mm})$ & $3.5 \pm 0.5$ & $3.5 \pm 0.6$ & $3.9 \pm 0.7$ & $>0.05$ \\
\hline LVESD (mm) & $3.1 \pm 0.7$ & $2.8 \pm 0.6$ & $5.0 \pm 0.9$ & $<0.001$ \\
\hline LVEDD (mm) & $5.0 \pm 0.7$ & $4.8 \pm 0.7$ & $6.1 \pm 0.9$ & 0.001 \\
\hline LVEF (\%) & $71.7 \pm 9.6$ & $76.5 \pm 9.1$ & $36.3 \pm 11.4$ & $<0.001$ \\
\hline Apical aneurysm (\%) & $7.7 \%$ & $25.0 \%$ & $18.2 \%$ & $>0.05$ \\
\hline \multicolumn{5}{|l|}{ 24-h ambulatory electrocardiogram } \\
\hline Isolated PVC (count/24 h) & $2029 \pm 4116$ & $602 \pm 1008$ & $7505 \pm 8246$ & 0.002 \\
\hline Couplets (count/24 h) & $16 \pm 39$ & $11 \pm 25$ & $457 \pm 730$ & $<0.001$ \\
\hline Polymorphic PVC (\%) & $50.0 \%$ & $50.0 \%$ & $91.7 \%$ & $>0.05$ \\
\hline NSVT (\%) & $14.3 \%$ & $12.5 \%$ & $58.3 \%$ & 0.006 \\
\hline \multicolumn{5}{|l|}{ Signal-averaged electrocardiogram } \\
\hline Duration (ms) & $92.6 \pm 7.0$ & $137.4 \pm 23.0$ & $140.7 \pm 32.7$ & $<0.001$ \\
\hline RMS40 $(\mu \mathrm{V})$ & $51.1 \pm 40.9$ & $17.4 \pm 8.9$ & $27.4 \pm 31.7$ & $<0.001$ \\
\hline LAS40 (ms) & $29.0 \pm 6.7$ & $47.6 \pm 20.1$ & $46.3 \pm 15.5$ & 0.002 \\
\hline Time domain positive (\%) & $14.3 \%$ & $45.8 \%$ & $41.6 \%$ & $>0.05$ \\
\hline MSC (\%) & $0.96 \pm 0.01$ & $0.95 \pm 0.01$ & $0.95 \pm 0.02$ & 0.01 \\
\hline SSC (\%) & $0.05 \pm 0.02$ & $0.07 \pm 0.03$ & $0.07 \pm 0.03$ & $>0.05$ \\
\hline MET (Hz) & $73.9 \pm 4.2$ & $85.0 \pm 12.8$ & $90.1 \pm 14.2$ & 0.001 \\
\hline SET $(H z)$ & $27.1 \pm 4.3$ & $37.9 \pm 16.9$ & $40.2 \pm 14.2$ & 0.01 \\
\hline Frequency domain positive (\%) & $35.7 \%$ & $70.8 \%$ & $75.0 \%$ & $>0.05$ \\
\hline
\end{tabular}

Data are reported as mean \pm SD or as percent. NYHA $=$ New York Heart Association; $L A D=$ left atrial diameter; LVESD = left ventricular end systolic diameter; LVEDD = left ventricular end diastolic diameter; LVEF = left ventricular ejection fraction; PVC = premature ventricular contraction; NSVT = nonsustained ventricular tachycardia; Duration = duration of ventricular activation; RMS40 = root-mean-squared voltage of the last $40 \mathrm{~ms}$ of ventricular activation; LAS40 = duration of the potentials below $40 \mu \mathrm{V}$ in terminal region until the offset of ventricular activation; MSC = mean of the spectral correlation series; SSC = standard deviation of the spectral correlation series; MET = mean of the edge track series; SET = standard deviation of the edge

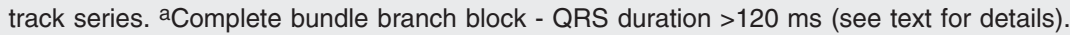

Categorial variables (\%) were analyzed statistically with the chi-square test and continuous variables (mean \pm $\mathrm{SD})$ were analyzed with Kruskal-Wallis ANOVA. 
when appropriate. Kruskal-Wallis ANOVA was used for inter-group comparison.

In univariate analyses, the relative risk (RR) for composite endpoint and respective 95\% confidence intervals $(\mathrm{CI})$ were assessed for all significant variables. For continuous variables, RR for adverse outcomes was estimated at an optimal dichotomization threshold calculated by receiver operating characteristic (ROC) curve analysis. In the latter, 24-h isolated PVC and couplet counts were assessed as continuous variables.

Variables found to be significant by univariate analysis were entered into a stepwise multivariate Cox proportional-hazards regression model to define independent predictors of the composite endpoint. The reproducibility of SAECG variables was assessed by $\kappa$ inter-rate agreement in two consecutive signal acquisitions in an independent group of 32 control volunteers (age, mean $\pm \mathrm{SD}, 58.5 \pm 11.0$ years, 6 females). Univariate $\alpha$ error level was set at 0.05 . In Cox proportional-hazard regression only variables with $\mathrm{P}<0.10$ in univariate analysis were entered into the model. The software packages used for data analysis were MS Excel 2000 (Microsoft Corporation, Redmond, WA, USA), Medcalc version 8 (Medcalc Sofware, Mariakerke, Belgium), Epi-Info 6.04 (Center of Disease Control, Atlanta, GA, USA), and Statgraphics version 5.1 Plus (Manugistic Inc., Rockville, MD, USA).

\section{Results}

\section{Clinical follow-up}

The overall mean follow-up was $84.2 \pm$ 39.0 months, ranging from 6 to 120 months, and was abbreviated by adverse events. Composite endpoint was observed in $34.0 \%$ (9 cardiac deaths and 11 episodes of VT, all nonsustained). According to the Los Andes classification on admission, adverse events were observed in $21.4 \%$ of subjects in group
1 (3 nonfatal), $20.8 \%$ in group 2 ( 1 fatal and 4 nonfatal) and in $75.0 \%$ in group 3 (8 fatal and 4 nonfatal; $\mathrm{P}=0.003$ ). At the end of follow-up, worsening in NYHA functional class was observed in $42 \%$ of the subjects (overall average reduction of NYHA functional class in 10 years: $1.71 \pm 1.01 ; \mathrm{P}<0.001$ ). Subjects who experienced adverse events showed a significant deterioration of NYHA functional class as compared to those who did not $(2.4 \pm 1.2 v s 1.3 \pm 0.5 ; \mathrm{P}=0.005)$. According to the Los Andes classification, worsening in NYHA functional class was observed in $33 \%$ of subjects in group 1, in $23 \%$ in group 2 , and in $92 \%$ in group $3(\mathrm{P}=$ 0.0004).

Reduction of LVEF was observed in $60.6 \%$ of subjects (overall average reduction of LVEF: $14.6 \pm 10.4 \%$; $\mathrm{P}<0.001)$, representing an average reduction of $4.3 \pm 4.6 \%$ per year $(\mathrm{P}<0.001)$. The average annual drop in ejection fraction in subjects who experienced adverse events during followup was not significantly different from the annual drop of those who did not experience adverse events $(2.8 \pm 3.3$ vs $2.6 \pm 4.8 \%$, respectively; $\mathrm{P}=0.54)$. According to the Los Andes classification, average annual reduction of LVEF was $1.3 \pm 2.4 \%$ in group $1,3.3$ $\pm 5.0 \%$ in group 2 , and $2.0 \pm 2.5 \%$ in group $3(\mathrm{P}=0.30)$.

At the end of follow-up, $3.4 \pm 0.6$ echocardiograms and $2.2 \pm 0.624$-h ambulatory ECG monitoring per subject had been analyzed. The overall average time taken from the first to the last exam assessed was reduced among subjects who experienced adverse events $(75.6 \pm 34.8$ months for subjects with no adverse events vs $27.6 \pm 27.6$ months for subjects with adverse events; $\mathrm{P}<$ 0.001). According to the Los Andes classification upon admission, no significant differences were observed regarding requests of echocardiograms (group $1=3.5 \pm 0.7$, group $2=3.5 \pm 0.5$, group $3=3.0 \pm 0.6 ; \mathrm{P}=$ $0.10)$ or 24-h ambulatory ECG monitoring (group $1=2.2 \pm 0.4$, group $2=2.3 \pm 0.6$, 
group $3=2.0 \pm 0.6 ; \mathrm{P}=0.22$ ) by primary assistance during follow-up. According to outcomes at the end of follow-up, no significant differences were observed in requests of echocardiograms $(3.5 \pm 0.6$ in the absence of adverse events vs $3.2 \pm 0.6$ in the presence of adverse events; $\mathrm{P}=0.11$ ) or 24 $\mathrm{h}$ ambulatory ECG monitoring $(2.3 \pm 0.5$ in the absence of adverse events vs $2.0 \pm 0.6$ in the presence of adverse event; $\mathrm{P}=0.07$ ) by primary assistance.

\section{Univariate analyses of clinical, resting 12-lead ECG, echocardiogram, 24-h ambulatory ECG monitoring, and signal-averaged ECG as predictors of composite endpoint}

Univariate analyses of significant variables of 12-lead resting ECG, echocardiogram, 24-h ambulatory ECG monitoring, and SAECG parameters are summarized in Table 2. The distribution of hypertensive subjects did not differ significantly between those who experienced (33.3\%) and did not experience $(45.2 \% ; \mathrm{P}=0.71)$ adverse events during follow-up. Age was not a significant predictor of adverse outcome $(\mathrm{P}=0.50)$.

Significant variables in univariate analysis for unfavorable outcome of composite endpoint were: i) demographic and clinical parameters: male gender $(\mathrm{RR}=2.6 ; 95 \% \mathrm{CI}$ $=1.3-5.5 ; \mathrm{P}=0.027), \mathrm{NYHA}>1(\mathrm{RR}=3.5$; $95 \% \mathrm{CI}=2.2-5.6 ; \mathrm{P}=0.01)$ and Los Andes class III $(\mathrm{RR}=3.6 ; 95 \% \mathrm{CI}=1.8-7.2 ; \mathrm{P}=$ $0.003)$; ii) echocardiographic parameters: apical aneurysm $(\mathrm{RR}=3.0 ; 95 \% \mathrm{CI}=1.6$ $5.7 ; \mathrm{P}=0.006), \mathrm{LVESD}(\mathrm{P}<0.001), \mathrm{LVEDD}$ $(\mathrm{P}<0.004)$, and LVEF $(\mathrm{P}<0.001)$, iii $) 12-$ lead resting ECG parameters: pathologic $\mathrm{Q}$ wave in 12-lead surface ECG $(\mathrm{RR}=2.5$; $95 \% \mathrm{CI}=1.2-5.0 ; \mathrm{P}=0.03)$; iv) $24-\mathrm{h}$ ambulatory ECG parameters: isolated-PVC counts/ $24 \mathrm{~h}(\mathrm{P}<0.001)$, couplet counts/24 h $(\mathrm{P}<$ $0.001)$, polymorphic-PVC $(\mathrm{RR}=4.1 ; 95 \%$ $\mathrm{CI}=1.1-16.4 ; \mathrm{P}=0.01)$, and $\mathrm{VT}(\mathrm{RR}=3.6$; $95 \% \mathrm{CI}=1.8-7.2 ; \mathrm{P}=0.001) ; \mathrm{v}) \mathrm{SAECG}$
Table 2. Clinical, electrocardiographic, and echocardiographic parameters on admission as a function of clinical outcomes during follow-up.

\begin{tabular}{|c|c|c|c|}
\hline & $\begin{array}{c}\text { Adverse } \\
\text { events }(N=17)\end{array}$ & $\begin{array}{c}\text { No adverse } \\
\text { events }(N=33)\end{array}$ & $\mathrm{P}$ \\
\hline \multicolumn{4}{|l|}{ Clinical variables } \\
\hline Age (years) & $51.5 \pm 11.1$ & $51.3 \pm 8.6$ & $>0.05$ \\
\hline Gender (F/M) & $8 / 9$ & $25 / 6$ & 0.03 \\
\hline NYHA functional class $>1(\%)$ & $23.5 \%$ & $0 \%$ & 0.01 \\
\hline \multicolumn{4}{|l|}{ Resting 12-lead electrocardiogram } \\
\hline P-wave duration (ms) & $87.6 \pm 21.9$ & $91.8 \pm 16.1$ & $>0.05$ \\
\hline QRS duration (ms) & $116.5 \pm 32.0$ & $110.9 \pm 30.8$ & $>0.05$ \\
\hline Left atrial overload (\%) & $17.7 \%$ & $9.1 \%$ & $>0.05$ \\
\hline Left bundle branch block (\%) ${ }^{\mathrm{a}}$ & $17.7 \%$ & $9.1 \%$ & $>0.05$ \\
\hline Right bundle branch block (\%)a & $35.3 \%$ & $48.5 \%$ & $>0.05$ \\
\hline Left anterior hemi-block (\%) & $41.2 \%$ & $27.3 \%$ & $>0.05$ \\
\hline Inactive area (\%) & $41.2 \%$ & $12.1 \%$ & 0.03 \\
\hline \multicolumn{4}{|c|}{ M-mode and 2-D mode echocardiogram } \\
\hline LAD (mm) & $3.8 \pm 0.7$ & $3.5 \pm 0.5$ & $>0.05$ \\
\hline LVESD (mm) & $4.1 \pm 1.1$ & $3.0 \pm 0.9$ & $<0.001$ \\
\hline LVEDD (mm) & $5.6 \pm 0.8$ & $4.9 \pm 0.8$ & 0.004 \\
\hline LVEF (\%) & $52.5 \pm 20.8$ & $73.8 \pm 12.5$ & $<0.001$ \\
\hline Apical aneurysm (\%) & $41.2 \%$ & $6.5 \%$ & 0.006 \\
\hline \multicolumn{4}{|l|}{ 24-h ambulatory electrocardiogram } \\
\hline Isolated PVC (count/24 h) & $5792 \pm 6963$ & $1043 \pm 3316$ & $<0.001$ \\
\hline Couplets (count/24 h) & $351 \pm 653$ & $11 \pm 39$ & $<0.001$ \\
\hline Polymorphic PVC (\%) & $88.2 \%$ & $51.5 \%$ & 0.01 \\
\hline NSVT (\%) & $52.9 \%$ & $9.1 \%$ & 0.001 \\
\hline \multicolumn{4}{|l|}{ Signal-averaged electrocardiogram } \\
\hline Duration (ms) & $134.2 \pm 31.9$ & $121.0 \pm 28.7$ & $>0.05$ \\
\hline RMS40 $(\mu \mathrm{V})$ & $23.6 \pm 25.1$ & $31.5 \pm 31.8$ & $>0.05$ \\
\hline LAS40 (ms) & $46.1 \pm 18.6$ & $40.1 \pm 17.9$ & $>0.05$ \\
\hline Time domain positive (\%) & $37.5 \%$ & $35.3 \%$ & $>0.05$ \\
\hline MSC (\%) & $0.95 \pm 0.02$ & $0.95 \pm 0.01$ & $>0.05$ \\
\hline SSC (\%) & $0.07 \pm 0.03$ & $0.06 \pm 0.02$ & $>0.05$ \\
\hline MET $(\mathrm{Hz})$ & $84.6 \pm 16.2$ & $82.0 \pm 10.7$ & $>0.05$ \\
\hline SET $(\mathrm{Hz})$ & $35.9 \pm 13.6$ & $35.0 \pm 15.4$ & $>0.05$ \\
\hline Frequency domain positive (\%) & $62.5 \%$ & $61.8 \%$ & $>0.05$ \\
\hline
\end{tabular}

Data are reported as mean \pm SD or as pecent. NYHA $=$ New York Heart Association; $\mathrm{LAD}=$ left atrial diameter; LVESD = left ventricular end systolic diameter; LVEDD = left ventricular end diastolic diameter; LVEF = left ventricular ejection fraction; PVC = premature ventricular contraction; NSVT = nonsustained ventricular tachycardia; Duration $=$ duration of the ventricular activation; RMS40 = root-mean-squared voltage of the last $40 \mathrm{~ms}$ of ventricular activation; LAS40 = duration of the potentials below $40 \mu \mathrm{V}$ in the terminal region until the offset of ventricular activation; $\mathrm{MSC}=$ mean of the spectral correlation series; SSC = standard deviation of the spectral correlation series; MET = mean of the edge track series; SET = standard deviation of the edge track series. aComplete bundle branch block - QRS duration >120 ms (see text for details).

The Student $t$-test or Mann-Whitney test was used to analyze continuous variables (mean $\pm \mathrm{SD}$ ) and the chi-square test or Fisher's exact test to analyze categorial variables $(\%)$, when appropriate. 
parameters: SAECG in time or frequency domains demonstrated significant agreement $(\kappa=0.92 ; 95 \% \mathrm{CI}=0.77-1.07$, and $\kappa=0.80$; $95 \% \mathrm{CI}=0.59-1.02$, respectively), in two consecutive acquisitions. SAECG parameters were nonsignificant predictors of composite endpoint or worsening of NYHA functional class or systolic function during follow-up.

The optimal thresholds of significant parameters assessed by ROC curve analysis were: LVESD > $2.8 \mathrm{~mm}(100.0 \%$ sensitivity, $56.2 \%$ specificity; $\mathrm{RR}=2.3 ; 95 \% \mathrm{CI}=1.5$ 3.4), LVEDD $>4.9 \mathrm{~mm}(81.2 \%$ sensitivity, $62.5 \%$ specificity; $\mathrm{RR}=2.2 ; 95 \% \mathrm{CI}=1.3$ 3.6), and LVEF <62\% (56.2\% sensitivity, $81.2 \%$ specificity; $\mathrm{RR}=3.2 ; 95 \% \mathrm{CI}=1.4-$ 7.4), isolated-PVC counts/24 h >614 (82.4\% sensitivity, $84.8 \%$ specificity; $R R=7.0$; $95 \% \mathrm{CI}=2.3-21.3)$ and couplet counts $/ 24 \mathrm{~h}$ $>6(70.6 \%$ sensitivity, $90.9 \%$ specificity; $\mathrm{RR}=5.1 ; 95 \% \mathrm{CI}=2.2-11.8$ ).

Multivariate analysis for composite endpoints

Multivariate analysis is summarized in Table 3. In multivariate analysis, significant univariate predictors (Los Andes class III, apical aneurysm, LVESD $>2.8 \mathrm{~mm}, \mathrm{LVEDD}$ $>4.9 \mathrm{~mm}, \mathrm{LVEF}<62 \%$, isolated-PVC counts/ $24 \mathrm{~h}>614$, couplet counts/24 h $>6$ and VT) were entered in a stepwise Cox-hazard regression model. After adjustment, the model converged (maximum likelihood ratio $=27.3$; 3 d.f.; $\mathrm{P}<0.001$ ), and the following variables were significant: apical aneurysm (haz-

Table 3. Multivariate Cox proportional-hazard analysis of adverse events during follow-up.

\begin{tabular}{lcccccc}
\hline Variable & B coefficient & SEM & z value & $P$ & $H R$ & $95 \% \mathrm{Cl}$ \\
\hline Apical aneurysm & 1.32 & 0.57 & 2.32 & 0.020 & 3.74 & $1.23-11.37$ \\
Isolated PVC count/24 $\mathrm{h}>614$ & 1.81 & 0.67 & 2.73 & 0.006 & 6.14 & $1.67-22.64$ \\
LVEF <62\% & 1.53 & 0.61 & 2.51 & 0.012 & 4.60 & $1.39-15.24$
\end{tabular}

SEM = standard error of the mean estimate; $\mathrm{HR}=$ hazard ratio; $95 \% \mathrm{Cl}=$ confidence interval at $95 \%$; PVC = premature ventricular contraction; LVEF = left ventricular ejection fraction. Cut-off thresholds determined by receiver operating characteristic analysis (see text for details). ard ratio $(\mathrm{HR})=3.7 ; 95 \% \mathrm{CI}=1.2-11.4 ; \mathrm{P}=$ $0.02)$, isolated-PVC count $/ 24 \mathrm{~h}>614(\mathrm{HR}=$ $6.1 ; 95 \% \mathrm{CI}=1.7-22.6 ; \mathrm{P}=0.006)$, and $\mathrm{LVEF}<62 \%(\mathrm{HR}=4.60 ; 95 \% \mathrm{CI}=1.4-$ $15.2 ; \mathrm{P}=0.01$ ) were independent predictors of adverse outcomes. The Kaplan-Meier curves for independent predictors are illustrated in Figure 1A-C.

\section{Discussion}

The objective of the present study was to assess clinical, electrocardiographic and echocardiographic parameters as predictors of adverse outcome during long-term follow-up of subjects with Chagas' disease staged according to the Los Andes classification upon admission. Among subjects with Chagas' disease assisted at our tertiary hospital outpatient clinics from 1995 and 1999, those with complaints of palpitations, corresponding to $9.1 \%$, and with normal atrialventricular conduction composed the cohort of the present study. Palpitations have been considered a harbinger of adverse outcome (26). On the other hand, conduction delay, reported in 5.8 to $20.2 \%$ of subjects with chronic Chagas' cardiomyopathy $(27,28)$, is a muscarinic-like immunoglobulin G-mediated disturbance considered to be a key point in the physiopathogenesis of intraventricular electrical abnormalities in Chagas' disease (29-32). The exclusion of subjects with atrioventricular delay at enrollment eliminated those at a higher risk for adverse events (11). Additionally, on admission, the use of nitroderivatives in Chagas' disease was not a consensus in our hospital due the lack of proved beneficial effects of these compounds. In fact, nitroderivatives have demonstrated conflicting results regarding eradication of parasitemia or retardation of the progression of Chagas' cardiomyopathy $(33,34)$. Recently, however, in a large nonrandomizedcontrolled trial, Viotti et al. (35) demonstrated a beneficial effect of benzimidazole in reducing the progression of Chagas' dis- 
ease in seropositive subjects in the indeterminate phase. These results await confirmation in large randomized controlled studies.

At the end of follow-up, the overall number of echocardiograms and 24-h ambulatory ECG monitoring requested per subject at the discretion of primary assistance did not demonstrate significant differences across the Los Andes classification groups or outcomes. However, a trend toward an increasing rate of request of exams for subjects who experienced more clinical complaints or worsening of clinical conditions during follow-up was observed, probably reflecting a concern of primary assistance regarding appropriate medical care delivery to these subjects.

In the present study, a composite endpoint of cardiac death and ventricular tachycardia was observed in $34.0 \%$ of subjects during follow-up, with an $18.0 \%$ total cardiac mortality rate (either sudden death or end-stage congestive heart failure). Adverse events were significantly more prevalent in group $3(75 \%)$ than in group $1(21.4 \%)$ and group $2(20.8 \%, \mathrm{P}=0.003)$, clearly representing the impact of the severity of cardiac involvement on prognosis at initial evaluation. Although the mortality rate in longterm follow-up studies varies in the literature, several factors seem to influence outcome, including the severity of systolic dysfunction and intraventricular conduction disturbances. In this context, studies by Espinosa et al. (7), Pimenta et al. (11), Hagar and Rahimtoola (31), and Bestetti and Muccillo (36) have reported 42, 36.6, 78, and 23\% mortality rates after 10 years of follow-up, respectively. Viotti et al. (34) assessed during a 10-year follow-up a large cohort of subjects with Chagas' disease who had been classified at admission according to the severity of cardiac involvement. The authors reported a $13 \%$ mortality rate in subjects with systolic dysfunction, and less than $1 \%$ in subjects with mild or no overt cardiac involvement.

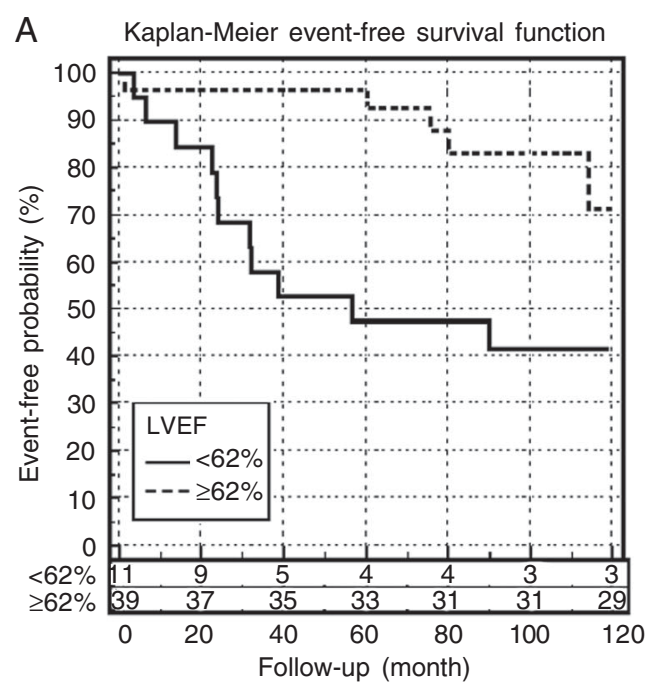

B Kaplan-Meier event-free survival function
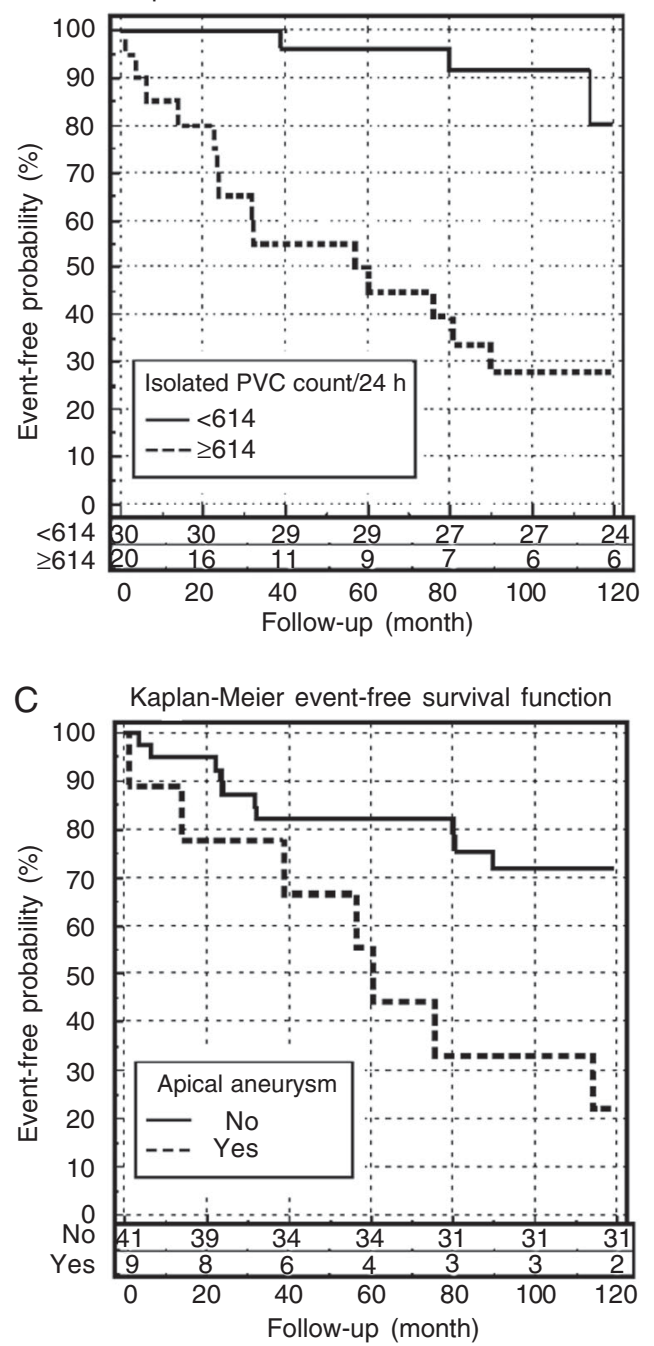

Figure 1. A, Kaplan-Meier eventfree probability function for adverse outcomes (composite endpoint) in subjects grouped according to left ventricular ejection fraction (LVEF) in the echocardiogram. LVEF threshold was assessed by analysis of receiver operating characteristic (ROC) curves. Note the significant increase in hazard ratio (HR) for adverse outcome toward the lower LVEF (HR = 4.60; $95 \% \mathrm{Cl}=1.39-15.24 ; \mathrm{P}=$ 0.012 , Cox proportional-hazard ' $z$ ' variable test). $B$, KaplanMeier event-free probability function for adverse outcomes (composite endpoint) in subjects grouped according to isolated premature ventricular contractions (PVC) count in 24-h ambulatory ECG ((isolated PVC count/24 h $<614$ or $\geq 614$ ) in ambulatory electrocardiogram. Isolated PVC threshold was assessed by analysis of ROC curves. Note the increased HR for adverse outcome toward the higher isolated PVC count (HR $=6.1 ; 95 \% \mathrm{Cl}=1.7-22.6 ; \mathrm{P}=$ 0.006, Cox proportional-hazard ' $z$ ' variable test). C, KaplanMeier event-free probability function for adverse outcomes (composite endpoint) in subjects grouped according to presence of apical aneurysm. Note a significant increase in $\mathrm{HR}$ for adverse outcome toward the higher isolated PVC count (HR $=3.74 ; 95 \% \mathrm{Cl}=1.23-11.37 ; \mathrm{P}$ $=0.020$, Cox proportional-hazard ' $z$ ' variable test). 
An important finding in the present study was that although LVEF on admission was an independent prognostic marker for adverse events, paradoxically, progression of left ventricular function (regardless of the Los Andes group or left ventricular function on admission), as assessed by LVEF during follow-up, did not differ between those who experienced adverse events and those who did not, assessed as composite endpoint. On the other hand, subjects who experienced adverse events developed a more rapid worsening of NYHA functional class when compared to those who did not experience adverse events. Thus, progression of ventricular dysfunction seems to be relentless and occupies a core position in Chagas' pathophysiology, although, by itself, it may not necessarily represent unfavorable evolution in a 10-year follow-up. Rather, worsening of NYHA function class faithfully reflected the evolution of the disease. Thus, it is attractive to speculate that, once installed, Chagas' heart disease inexorably progresses, but it is the cardiovascular status determined in turn by the immune response at early stages of infection that actually influences prognosis.

In the present study, after optimal dichotomization, male gender, NYHA $>1$, apical aneurysm, LVESD $>2.8 \mathrm{~mm}$, LVEDD $>4.9 \mathrm{~mm}, \mathrm{LVEF}<62 \%$, isolated-PVC counts/ $24 \mathrm{~h}>614$, couplet counts $/ 24 \mathrm{~h}>6$, and previous documented episodes of nonsustained VT assessed on admission were univariate prognostic markers of a composite of cardiac mortality and ventricular tachycardia endpoints (Table 2). These findings as predictors of cardiac mortality and arrhythmic events agree with reports by other investigators (7-10,14,37).

In particular, concerning SAECG analysis in the time and frequency domains, the diagnostic results showed appropriate reproducibility, and abnormal values were directly associated with growing complexity of heart disease according to the Los Andes classification. Dopico et al. (38) have previously reported similar findings limited to time domain analysis (Table 1). In other words, in Chagas' disease, electrical markers of myocardial fibrosis are directly associated with the severity of cardiac involvement (39). Presently, an inactive area in 12lead ECG was associated with longer late potentials (duration of the potentials below $40 \mu \mathrm{V}$ in terminal region until the offset of ventricular activation: $53.3 \pm 18.5 \mathrm{~ms}$, inactive areas, vs $39.0 \pm 17.0 \mathrm{~ms}$, no inactive areas; $\mathrm{P}=0.02$ ) and higher turbulence (mean of the edge track time-series: $90.4 \pm 12.3$ $\mathrm{Hz}$, inactive areas, vs $80.8 \pm 12.2 \mathrm{~Hz}$, no inactive areas; $\mathrm{P}=0.03)$. Nevertheless, SAECG failed to demonstrate statistically significant prognostic value for composite endpoint during follow-up. Although a very small number of long-term follow-up studies have been conducted to assess SAECG as a predictor of cardiac mortality in Chagas' disease, conflicting results still arise from studies assessing the role of SAECG in predicting arrhythmic events $(24,40)$. In the present study, SAECG parameters did show a significant predictive value for arrhythmic events during follow-up (data not shown). The explanations for these findings are still elusive. One can speculate that autonomic dysfunction and inflammation (34) when superimposed to myocardial fibrosis play key roles in the mechanism of cardiac death.

Finally, in the present study, apical aneurysm, LVEF and PVC/24 h were independent predictors of a composite of cardiac death and ventricular arrhythmia. Accordingly, echocardiography and 24-ambulatory ECG monitoring are germane in the admission workup of subjects with Chagas' disease.

\section{Limitations of the study}

A potential limitation of this investigation is that it was performed on a hospitalderived cohort, perhaps restricting the extension of the present findings to a general, 
unselected population of chagasic subjects.

In the present study, optimal normality thresholds for continuous variables were defined by ROC analyses. Optimal cut-off values for LVEF and LVESD were respectively higher and lower than conventionally defined thresholds for other forms of cardiomyopathy. A direct extension of the current findings to clinical practice still needs to be confirmed.

Sustained VT was not documented among survivors during follow-up. This may represent a limitation, since subjects who died suddenly may have experienced fatal ventricular arrhythmias, which were not documented as sustained VT.

Overall data analysis of significant variables achieved high statistical power $(>90 \%)$ in post hoc sampling analysis, validating the present results. However, some variables may lack statistical significance due to the need for specific Chagas' subpopulationoriented studies.

We conclude that clinical (male gender and NYHA functional class), electrocardiographic (pathologic Q wave on a 12-lead ECG, frequent and polymorphic PVC and VT episodes during 24-h ambulatory monitoring), and echocardiographic (apical an- eurysm, left ventricular end systolic and diastolic diameters and ejection fraction) parameters are markers of unfavorable prognosis in subjects with Chagas' disease. In particular, apical aneurysms, reduced left ventricular ejection fraction, and frequent ventricular premature complexes in 24-h ambulatory ECG are independent predictors of a composite of cardiac mortality and ventricular tachycardia during follow-up. Signal-averaged electrocardiogram is useful to reveal electrical disturbances due to myocardial fibrosis, although it demonstrated no prognostic value for the endpoint investigated.

\section{Acknowledgments}

We are grateful to Prof. José Barbosa Filho, Universidade Gama Filho and Instituto Nacional de Cardiologia Laranjeiras, for his suggestions and support in revising this manuscript, to Dr. Eduardo Correa Barbosa and Dr. Alfredo de Souza Bomfim, Universidade do Estado do Rio de Janeiro, for their insights on data analysis, and to Dr. Adriano de Moraes, for support in carrying out signal-averaged ECG and 24-h ambulatory ECG exams on admission.

\section{References}

1. Marin Neto JA, Simoes MV, Sarabanda AV. Chagas' heart disease. Arq Bras Cardiol 1999; 72: 247-280.

2. Guide of Diseases Page. Brazilian Federal Government, Official Report on Chagas' Disease. http://www.pgr.mpf.gov.br/pgr/saude/ doencas/chagas.htm. Accessed April 13, 2006.

3. Vinhaes MC, Carlos ALF. Epidemiological Status of Infective Diseases in Brazil. Annual Health Ministry Report. http://dtr2001.saude. gov.br/svs/epi/situacao_doencas/situacao.htm\#chagas. Accessed April 13, 2006.

4. Hagar JM, Rahimtoola SH. Chagas' heart disease. Curr Probl Cardiol 1995; 20: 825-924.

5. Maguire JH, Hoff R, Sherlock I, Guimaraes AC, Sleigh AC, Ramos NB, et al. Cardiac morbidity and mortality due to Chagas' disease: prospective electrocardiographic study of a Brazilian community. Circulation 1987; 75: 1140-1145.

6. Gazin P, Melo G, Abuquerque A, Oliveira W Jr, Soula G, Audry P. Chagas disease in a rural area of Northeast Brazil. Bull Soc Pathol Exot 2004; 97: 189-192.
7. Espinosa RA, Pericchi LR, Carrasco HA, Escalante A, Martinez O, Gonzalez R. Prognostic indicators of chronic chagasic cardiopathy. Int J Cardiol 1991; 30: 195-202.

8. Carrasco HA, Parada H, Guerrero L, Duque M, Duran D, Molina C. Prognostic implications of clinical, electrocardiographic and hemodynamic findings in chronic Chagas' disease. Int J Cardiol 1994; 43: 27-38.

9. Bestetti RB, Dalbo CM, Freitas OC, Teno LA, Castilho OT, Oliveira JS. Noninvasive predictors of mortality for patients with Chagas' heart disease: a multivariate stepwise logistic regression study. Cardiology 1994; 84: 261-267.

10. Bestetti RB, Dalbo CM, Arruda CA, Correia FD, Freitas OC. Predictors of sudden cardiac death for patients with Chagas' disease: a hospital-derived cohort study. Cardiology 1996; 87: 481-487.

11. Pimenta J, Valente N, Miranda M. Long-term follow up of asymptomatic chagasic individuals with intraventricular conduction disturbances, correlating with non-chagasic patients. Rev Soc Bras Med Trop 1999; 32: 621-631. 
12. Ribeiro AL, Rocha MO, Barros MV, Rodrigues AR, Machado FS. A narrow QRS does not predict a normal left ventricular function in Chagas' disease. Pacing Clin Electrophysiol 2000; 23: 2014-2017.

13. Lux RL, Hilbel T, Brockmeier K. Electrocardiographic measures of repolarization revisited: why? what? how? J Electrocardiol 2001; 34 (Suppl): 259-264.

14. Salles G, Xavier S, Sousa A, Hasslocher-Moreno A, Cardoso C. Prognostic value of QT interval parameters for mortality risk stratification in Chagas' disease: results of a long-term follow-up study. Circulation 2003; 108: 305-312.

15. Anonymous. Signal-averaged electrocardiography. J Am Coll Cardiol 1996; 27: 238-249.

16. Bailey JJ, Berson AS, Handelsman H, Hodges M. Utility of current risk stratification tests for predicting major arrhythmic events after myocardial infarction. J Am Coll Cardiol 2001; 38: 1902-1911.

17. Goedel-Meinen L, Hofmann M, Ryba S, Schomig A. Prognostic value of an abnormal signal-averaged electrocardiogram in patients with nonischemic dilated cardiomyopathy. Am J Cardiol 2001; 87: 809-812.

18. Vallejo M, Reyes K, Reyes PA, Gonzalez Hermosillo JA. Late potentials and variability of cardiac frequency in chronic chagasic myocardiopathy and other myocardiopathies. Arch Inst Cardiol Mex 1997; 67: 485-493.

19. Mora F, Gomis P, Passariello G. High resolution ECG signals in Chagas patients: SEARCH project. Acta Cient Venez 1999; 50: $187-$ 194.

20. Schocken DD, Arrieta MI, Leaverton PE, Ross EA. Prevalence and mortality rate of congestive heart failure in the United States. $J A m$ Coll Cardiol 1992; 20: 301-306.

21. Acquatella H, Schiller NB, Puigbo JJ, Giordano H, Suarez JA, Casal $\mathrm{H}$, et al. M-mode and two-dimensional echocardiography in chronic Chagas' heart disease. A clinical and pathologic study. Circulation 1980; 62: 787-799.

22. Barbosa PR, Barbosa EC, Bomfim AS, Ginefra P, Nadal J. Clinical assessment of the effect of digital filtering on the detection of ventricular late potentials. Braz J Med Biol Res 2002; 35: 1285-1292.

23. Barbosa PR, Sousa MO, Barbosa EC, Bomfim AS, Ginefra P, Nadal $\mathrm{J}$. Analysis of the prevalence of ventricular late potentials in the late phase of myocardial infarction based on the site of infarction. Arq Bras Cardiol 2002; 78: 352-363.

24. de Moraes AP, Moffa PJ, Sosa EA, Bellotti GM, Pastore CA, Lima $\mathrm{EV}$, et al. Signal-averaged electrocardiogram in chronic Chagas' heart disease. São Paulo Med J 1995; 113: 851-857.

25. Barbosa EC, Barbosa PR, Ginefra P, Albanesi Filho FM. High resolution electrocardiogram in frequency domain. Use of statistical techniques of spectral correlation for identification of patients with sustained monomorphic ventricular tachycardia. Arq Bras Cardiol 1998; 71: 595-599.

26. Bestetti RB, Freitas OC, Muccillo G, Oliveira JS. Clinical and morphological characteristics associated with sudden cardiac death in patients with Chagas' disease. Eur Heart J 1993; 14: 1610-1614.

27. Dias JC, Kloetzel K. The prognostic value of the electrocardiographic features of chronic Chagas' disease. Rev Inst Med Trop São Paulo 1968; 10: 158-162.

28. Maguire JH, Mott KE, Lehman JS, Hoff R, Muniz TM, Guimaraes $\mathrm{AC}$, et al. Relationship of electrocardiographic abnormalities and seropositivity to Trypanosoma cruzi within a rural community in northeast Brazil. Am Heart J 1983; 105: 287-294.

29. de Carvalho AC, Masuda MO, Tanowitz HB, Wittner M, Goldenberg RC, Spray DC. Conduction defects and arrhythmias in Chagas' disease: possible role of gap junctions and humoral mechanisms. $J$ Cardiovasc Electrophysiol 1994; 5: 686-698.

30. Costa PC, Fortes FS, Machado AB, Almeida NA, Olivares EL, Cabral PR, et al. Sera from chronic chagasic patients depress cardiac electrogenesis and conduction. Braz J Med Biol Res 2000; 33: 439-446.

31. Hagar JM, Rahimtoola SH. Chagas' heart disease in the United States. N Engl J Med 1991; 325: 763-768.

32. Medei M, Costa PC, Barbosa PR, Hernandez CC, Chaves EA, Linhares $\mathrm{V}$, et al. Human chagasic antibodies with muscarinic activity modulate ventricular repolarization: basis for electrical disturbance. Int J Cardiol 2006 (in press).

33. Lauria-Pires L, Braga MS, Vexenat AC, Nitz N, Simoes-Barbosa A, Tinoco DL, et al. Progressive chronic Chagas heart disease ten years after treatment with anti-Trypanosoma cruzi nitroderivatives. Am J Trop Med Hyg 2000; 63: 111-118.

34. Viotti R, Vigliano C, Lococo B, Petti M, Bertocchi G, Alvarez MG, et al. Clinical predictors of chronic chagasic myocarditis progression. Rev Esp Cardiol 2005; 58: 1037-1044.

35. Viotti R, Vigliano C, Lococo B, Bertocchi G, Petti M, Alvarez MG, et al. Long-term cardiac outcomes of treating chronic Chagas disease with benzimidazole versus no treatment: a nonrandomized trial. Ann Intern Med 2006; 144: 724-734.

36. Bestetti RB, Muccillo G. Clinical course of Chagas' heart disease: a comparison with dilated cardiomyopathy. Int J Cardiol 1997; 60: 187-193.

37. Acquatella H, Catalioti F, Gomez-Mancebo JR, Davalos V, Villalobos L. Long-term control of Chagas disease in Venezuela: effects on serologic findings, electrocardiographic abnormalities, and clinical outcome. Circulation 1987; 76: 556-562.

38. Dopico LR, Nadal J, Infantosi AFC. Analysis of late potentials in the high-resolution electrocardiogram of patients with Chagas' disease using weighted coherent average. Braz J Biomed Eng 2000; 16: 4959.

39. Bestetti RB. Predictors of unfavourable prognosis in chronic Chagas' disease. Trop Med Int Health 2001; 6: 476-483.

40. Fuenmayor AJ, Fuenmayor AM, Carrasco H, Parada H, Fuenmayor C, Jugo D. Results of electrophysiologic studies in patients with acute Chagasic myocarditis. Clin Cardiol 1997; 20: 1021-1024. 